\title{
Prediction of femtosecond oscillations in the transient current of a quantum dot in the Kondo regime
}

\author{
A. Goker, ${ }^{1,2}$ Z. Y. Zhu, ${ }^{2}$ A. Manchon, ${ }^{2}$ and U. Schwingenschlögl ${ }^{2}$ \\ ${ }^{1}$ Department of Physics, Bilecik University, 11210, Gulumba, Bilecik, Turkey \\ ${ }^{2}$ Materials Science \& Engineering, Physical Science \& Engineering Division, KAUST, 23955-6900 Thuwal, Kingdom of Saudi Arabia
}

(Received 29 July 2010; published 11 October 2010)

\begin{abstract}
We invoke the time-dependent noncrossing approximation in order to study the effects of the density of states of gold contacts on the instantaneous conductance of a single electron transistor which is abruptly moved into the Kondo regime by means of a gate voltage. For an asymmetrically coupled system, we observe that the instantaneous conductance in the Kondo time scale exhibits beating with distinct frequencies, which are proportional to the separation between the Fermi level and the sharp features in the density of states of gold. Increasing the ambient temperature or bias quenches the amplitude of the oscillations. We attribute the oscillations to interference between the emerging Kondo resonance and van-Hove singularities in the density of state. In addition, we propose an experimental realization of this model.
\end{abstract}

DOI: 10.1103/PhysRevB.82.161304

PACS number(s): 72.15.Qm, 85.35.-p

There is a growing need to provide a quantitative description of the sudden switching behavior of single electron transistors since the continuous shrinking of conventional metaloxide-semiconductor field-effect transistors ${ }^{1}$ and developments in state-of-the-art nanotechnology experiments suggest they may one day constitute building blocks of organic computers. The real-time electron dynamics in such devices have also profound implications for quantum computing ${ }^{2}$ and the realization of an electron analog of a single-photon gun. ${ }^{3}$

The effect of sudden perturbation in the form of steplike switching of the gate or bias voltage has been studied thoroughly ${ }^{4-7}$ and it has been unambiguously shown that the resulting transient current exhibits different time scales. ${ }^{8-11}$ The initial fast non-Kondo time scale is characterized by a reshaping of the broad Breit-Wigner resonance located around the impurity level whereas the sharp Kondo resonance pinned to the Fermi level reaches a metastable state at the end of the much longer Kondo time scale. Subsequent investigations predicted that the asymmetric coupling of the dot to the contacts may induce interference between the Kondo resonance and the discontinuities in the density of states (DOS) of the leads. ${ }^{12}$ Extension of the diagrammatic Monte Carlo method $^{13}$ to impurities out of equilibrium ${ }^{14}$ verified the transient current's dependency on the bandwidth of the contacts. ${ }^{15}$

Ramifications of the band structure of contacts in timedependent transport have been elucidated previously for prototypical systems by directly solving the Green's functions in the time domain. ${ }^{12,16,17} A b$ initio calculations also suggest that the crystallographic orientation of electrodes may influence transport properties. ${ }^{18}$

The purpose of this Rapid Communication is to provide a more realistic picture relevant to an actual experiment by using the real density of states of gold obtained from firstprinciples calculations as input in a study of the transient current through a quantum dot asymmetrically coupled to gold leads. The dot level is abruptly switched such that the Kondo resonance is present in the final state. We analyze the effects of the density of states of the conduction electrons in the contacts, the position of the dot energy level, the asym- metries in the couplings, and the temperature on the instantaneous current.

The physics of this system is captured sufficiently by a single impurity Anderson Hamiltonian which describes a single doubly degenerate level of energy $\epsilon_{d o t}$ attached to continuum electron baths. There is a one-to-one mapping between this model and the Kondo model via the SchrieferWolff transformation for the parameter range we consider this Rapid Communication. The auxiliary boson transformation is performed for the Anderson Hamiltonian. In this method, the electron operator on the impurity is replaced by a massless boson operator and a pseudofermion operator. The $U \rightarrow \infty$ limit is achieved by restricting the sum of the number of bosons and pseudofermions to unity. The Hamiltonian then converts into

$$
H(t)=\sum_{k \alpha \sigma}\left[\epsilon_{k} n_{k \alpha \sigma}+V_{\alpha}\left(\varepsilon_{k \alpha}, t\right) c_{k \alpha \sigma}^{\dagger} b^{\dagger} f_{\sigma}+\text { H.c. }\right]+\sum_{\sigma} \epsilon_{d o t}(t) n_{\sigma},
$$

where $f_{\sigma}^{\dagger}\left(f_{\sigma}\right)$ and $c_{k \alpha \sigma}^{\dagger}\left(c_{k \alpha \sigma}\right)$ with $\alpha=\mathrm{L}, \mathrm{R}$ create (annihilate) an electron of spin $\sigma$ in the dot and in the left (L) and right (R) gold leads, respectively. Moreover, $n_{\sigma}$ and $n_{k \alpha \sigma}$ are the corresponding number operators, $V_{\alpha}$ are the tunneling amplitudes for the left and the right leads, and $b^{\dagger}(b)$ creates (annihilates) a massless boson in the impurity.

Assuming that the hopping matrix elements have no explicit time dependency, the coupling of the quantum dot to the contacts can be parametrized as $\Gamma_{\mathrm{L}(\mathrm{R})}(\epsilon)=\bar{\Gamma}_{\mathrm{L}(\mathrm{R})} \xi_{\mathrm{L}(\mathrm{R})}(\epsilon)$, where $\bar{\Gamma}_{\mathrm{L}(\mathrm{R})}=2 \pi\left|V_{\mathrm{L}(\mathrm{R})}\left(\epsilon_{f}\right)\right|^{2}$ is a constant and $\xi_{\mathrm{L}(\mathrm{R})}(\epsilon)$ is the density-of-states function. In order to describe the density of states of the gold contacts accurately, we employ $a b$ initio calculations.

The $a b$ initio calculation of the gold density of states is based on density-functional theory. We make use of the wellestablished WIEN2K package, ${ }^{19,20}$ in which the full-potential linearized augmented plane-wave method is implemented with a dual-basis set. Specifically, the gold core states are defined by the electronic configuration $\mathrm{Kr} 4 d^{10} 4 f^{14} 5 s^{2}$, 


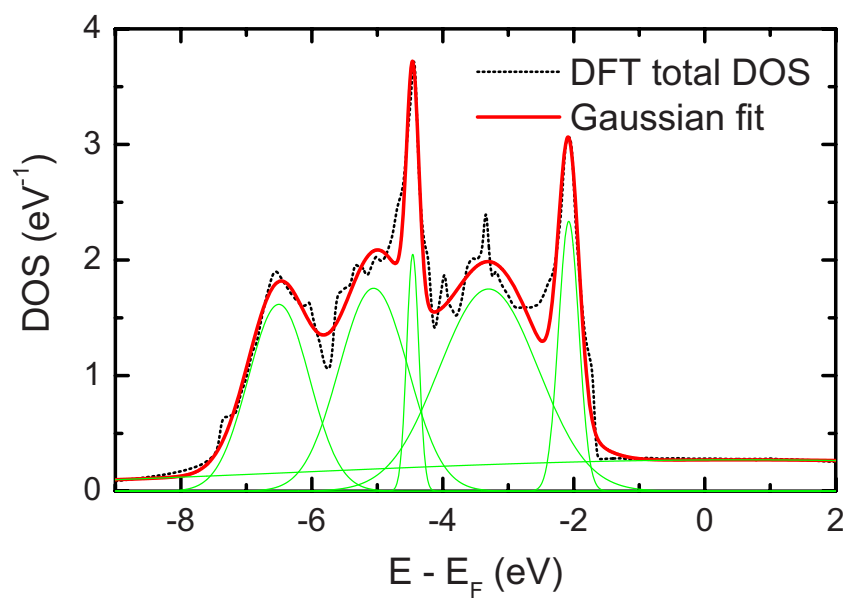

FIG. 1. (Color online) The total density of states of gold is shown by a black curve as a function of the separation from the Fermi level. The bold red curve corresponds to the best fit to the $a b$ initio data by a linear combination of Gaussian functions, which are represented by thin green curves.

whereas the valence states comprise $5 p, 5 d, 6 s$, and $6 p$ orbitals. The face-centered cubic unit cell of gold (space group $F m \overline{3} m$, lattice constant $a=4.080 \AA$ ) with four atoms per cell is used in the calculation. For the exchange-correlation potential the generalized gradient approximation within the Perdew, Burke, and Ernzerhof parametrization is adopted. ${ }^{21}$ The radius of the gold muffin-tin sphere is set to $2.5 \mathrm{Bohr}$ radii. Moreover, the plane-wave cutoff for the scalar relativistic basis function is given by $R_{m t} K_{\max }=8$ and $l_{\max }=10$. Integrations in the reciprocal space for self-consistent field cycles apply the tetrahedron method and $752 k$ points in the irreducible wedge of the Brillouin zone. The resulting DOS is shown in Fig. 1.

In order to represent this complicated curve in a functional form, we use a fitting procedure with a linear combination of Gaussian functions given by

$$
\rho(\epsilon)=\sum_{i} \rho_{i}(\epsilon),
$$

where

$$
\rho_{i}(\epsilon)=\frac{\alpha_{i}}{\zeta_{i} \sqrt{0.5 \pi}} \exp \left[-2\left(\frac{\epsilon-\epsilon_{i}}{\zeta_{i}}\right)^{2}\right] .
$$

The outcome of the fitting procedure, that involves six different Gaussians of varying linewidth and peak position, is also shown in Fig. 1. The number of Gaussians corresponds to the number of gold states in the vicinity of the Fermi level

TABLE I. Fitting parameters for six different Gaussians used to replicate the density of states of gold (in $\mathrm{eV}$ ).

\begin{tabular}{rrrrrrr}
\hline \hline & \multicolumn{1}{c}{1} & \multicolumn{1}{c}{2} & \multicolumn{1}{c}{3} & \multicolumn{1}{c}{4} & \multicolumn{1}{c}{5} & \multicolumn{1}{c}{6} \\
\hline$\epsilon_{i}$ & -6.503 & -5.057 & -4.459 & -3.300 & -2.077 & 0.557 \\
$\zeta_{i}$ & 0.934 & 1.055 & 0.185 & 1.513 & 0.310 & 13.474 \\
$\alpha_{i}$ & 1.892 & 2.321 & 0.475 & 3.317 & 0.907 & 4.580 \\
\hline \hline
\end{tabular}

(five $d$ orbitals and one $s$ orbital). The resulting best fit parameters are summarized in Table I. Throughout the rest of this Rapid Communication, we will switch to atomic units, where $\hbar=k_{B}=e=1$, and perform our calculations accordingly.

We invoke the well-tested noncrossing approximation (NCA) to obtain the pseudofermion and slave boson selfenergies. NCA gives reliable results for dynamical quantities except for very low temperatures or finite magnetic field. We stay away from both these regimes. We solve the resulting real-time coupled integrodifferential Dyson equations for the retarded and lesser Green's functions in a discrete twodimensional grid. A technical description of our implementation has been published elsewhere. ${ }^{11,22}$

The net current in the electrical circuit can be derived from the Green's functions $G_{p s e u}^{<\mathrm{R})}\left(t, t^{\prime}\right)$ and $B^{<(\mathrm{R})}\left(t, t^{\prime}\right)$. We will denote the net current by $I(t)=I_{\mathrm{L}}(t)-I_{\mathrm{R}}(t)$, where $I_{\mathrm{L}}(t)$ $\left[I_{\mathrm{R}}(t)\right]$ represents the net current from the left (right) contact through the left (right) barrier to the dot. The general expression for the net current ${ }^{23}$ can be rewritten using pseudofermion and slave boson Green's functions, ${ }^{12}$ finally leading to

$$
\begin{aligned}
I(t)= & -2\left(\bar{\Gamma}_{\mathrm{L}}-\bar{\Gamma}_{\mathrm{R}}\right) \operatorname{Re}\left[\int_{-\infty}^{t} d t_{1} \xi_{o}\left(t, t_{1}\right) h\left(t-t_{1}\right)\right] \\
& +2 \bar{\Gamma}_{\mathrm{L}} \operatorname{Re}\left\{\int_{-\infty}^{t} d t_{1}\left[\xi_{o}\left(t, t_{1}\right)+\xi_{u}\left(t, t_{1}\right)\right] f_{\mathrm{L}}\left(t-t_{1}\right)\right\} \\
& -2 \bar{\Gamma}_{\mathrm{R}} \operatorname{Re}\left\{\int_{-\infty}^{t} d t_{1}\left[\xi_{o}\left(t, t_{1}\right)+\xi_{u}\left(t, t_{1}\right)\right] f_{\mathrm{R}}\left(t-t_{1}\right)\right\}
\end{aligned}
$$

with $\xi_{o}\left(t, t_{1}\right)=G_{p s e u}^{<}\left(t, t_{1}\right) B^{\mathrm{R}}\left(t_{1}, t\right)$ as well as $\xi_{u}\left(t, t_{1}\right)$ $=G_{\text {pseu }}^{\mathrm{R}}\left(t, t_{1}\right) B^{<}\left(t_{1}, t\right)$. In Eq. (4), $f_{\mathrm{L}}\left(t-t_{1}\right)$ and $f_{\mathrm{R}}\left(t-t_{1}\right)$ are the convolutions of the density-of-states function with the Fermi-Dirac distributions of the left and right contacts, respectively, while $h\left(t-t_{1}\right)$ is the Fourier transform of the density of states. ${ }^{12}$ The conductance $G$ is given by the current divided by the bias voltage $V$. The subsequent timedependent conductance results are computed by Eq. (4). We will be referring to $\eta=\frac{\bar{\Gamma}_{\mathrm{L}}}{\bar{\Gamma}_{\text {tot }}}$, where $\bar{\Gamma}_{t o t}=\bar{\Gamma}_{\mathrm{L}}+\bar{\Gamma}_{\mathrm{R}}$, as the asymmetry factor.

The Kondo effect is a many-body resonance arising at low temperatures due to a spin singlet formed from the hybridization of the net free spin inside the dot with the continuum electrons in the contacts. It originates from the seminal work of Jun Kondo, ${ }^{24}$ where he discovered that a divergence in the perturbation series of tunnel couplings yields resistance enhancements in metals containing magnetic impurities. Its hallmark is a sharp resonance pinned to the Fermi levels of the contacts in the dot density of states. The broadening of the Kondo resonance is given by the energy scale (Kondo temperature)

$$
T_{K} \approx\left(\frac{D \Gamma_{t o t}}{4}\right)^{1 / 2} \exp \left(-\frac{\pi\left|\epsilon_{\mathrm{dot}}\right|}{\Gamma_{\text {tot }}}\right) .
$$

Here, $D$ is an energy cutoff, equal to half the bandwidth of the conduction electrons, and $\Gamma_{t o t}$ is the value of the total 


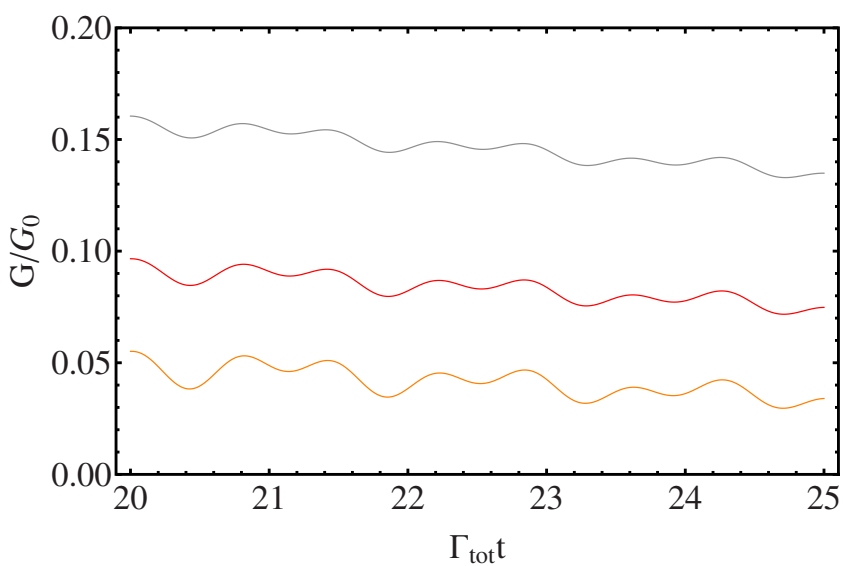

FIG. 2. (Color online) The gray, red, and orange curves (from top to bottom) show the instantaneous conductance versus time in the Kondo time scale after the dot level has been switched to its final position for asymmetry factors of $0.85,0.9$, and 0.95 , respectively, at $T=0.009 \Gamma_{\text {tot }}$ and $V=T_{K}$ for constant $\Gamma_{t o t}$.

coupling between the dot and the contacts $\Gamma_{t o t}(\epsilon)$ at $\epsilon=\epsilon_{F}$.

In the following, we investigate the instantaneous conductance for a system in which the dot level is abruptly shifted from $\epsilon_{1}=-5 \Gamma_{\text {tot }}$ to $\epsilon_{2}=-2 \Gamma_{\text {tot }}$, where $\Gamma_{t o t}=0.8 \mathrm{eV}$ at $t=0$ by a gate voltage, yielding a transition from a non-Kondo $\left(T_{K} \ll T\right)$ to a Kondo state. In the final state, we infer $T_{K}=0.0025 \Gamma_{\text {tot }}$ from Eq. (5). In the initial short-time scale associated with charge fluctuations, the conductance reaches a maximum for large asymmetry factors and then starts to decay, confirming previous studies. ${ }^{12}$ Figure 2 shows the behavior of the instantaneous conductance in the long Kondo time scale after the dot level has been switched to its final position. The instantaneous conductance exhibits a complex ringing behavior and the amplitude of the oscillations diminishes with decreasing asymmetry factor, completely disappearing for symmetric coupling. This is simply because the interference between the left contact and the Kondo resonance is out of phase with the interference between the right contact and the Kondo resonance due to opposite signs in Eq. (4). The amplitudes of these two interference processes are equal for symmetric coupling and the oscillations cancel out.

The frequencies taking part in the oscillations can be extracted by a Fourier transform of the time-dependent conductance. It turns out that there exist two distinct frequencies, $\omega_{1}$ and $\omega_{2}$, which give rise to a beating behavior with envelope and carrier frequencies of $\omega_{1}-\omega_{2}$ and $\omega_{1}+\omega_{2}$, respectively. We find $\omega_{1}=2.15 \omega_{2}$, which corresponds to the ratio of the distances of the peaks at -2.08 and $-4.46 \mathrm{eV}$ in Fig. 1 from the Fermi level. This result demonstrates that van-Hove singularities can be probed by the transient current in the Kondo regime.

The effect of finite bias on the instantaneous conductance is depicted in Fig. 3. The influence of finite bias is twofold. First, it reduces the amplitude of the oscillations. Second, the decay rate of the oscillations, defined as the inverse of the time it takes the oscillations to die out, increases, indicating that the oscillations are inherently related to the formation of the Kondo resonance. It should be noted that the split Kondo peak oscillations at finite bias have no discernible effect on

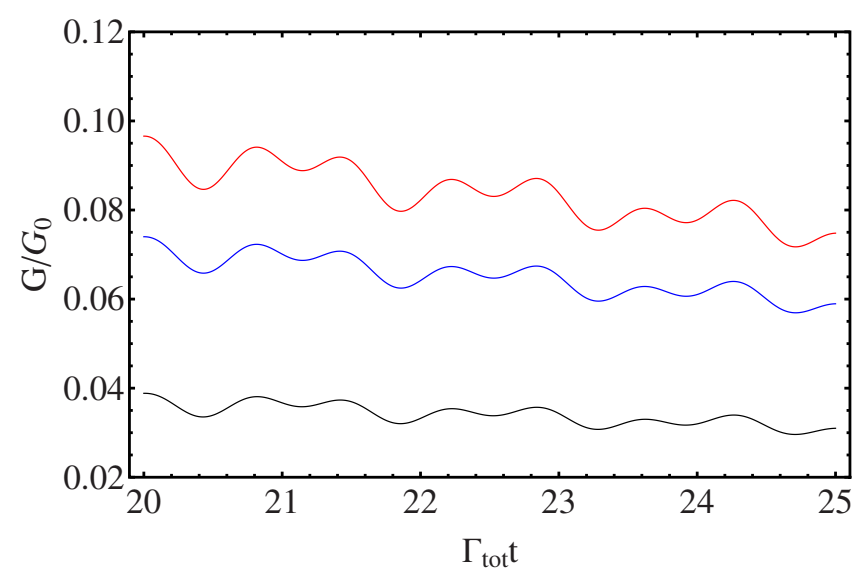

FIG. 3. (Color online) The red, blue, and black curves (from top to bottom) represent the instantaneous conductance versus time in the Kondo time scale after the dot level has been switched to its final position for an asymmetry factor of 0.9 at $T=0.009 \Gamma_{t o t}$ when the bias is equal to $V=T_{K}, V=4 T_{K}$, and $V=8 T_{K}$, respectively, for constant $\Gamma_{\text {tot }}$.

the results as their frequency is two orders of magnitude smaller.

Finally, the effect of ambient temperature is displayed in Fig. 4. Lowering the ambient temperature influences the time-dependent conductance results in the following ways. First, the decay rate of the oscillations decreases. Second, the amplitude of the oscillations starts increasing but saturates when the temperature approaches the Kondo temperature $T_{K}$. Lowering the temperature below $T_{K}$ does not alter the amplitude anymore.

Based on these observations and the fact that discontinuities in the density of states of the contacts can induce interference with the Kondo resonance for an asymmetrically coupled system, ${ }^{12}$ we suggest that the beating behavior in the time-dependent conductance is a result of interference between the emerging Kondo resonance at the Fermi level and

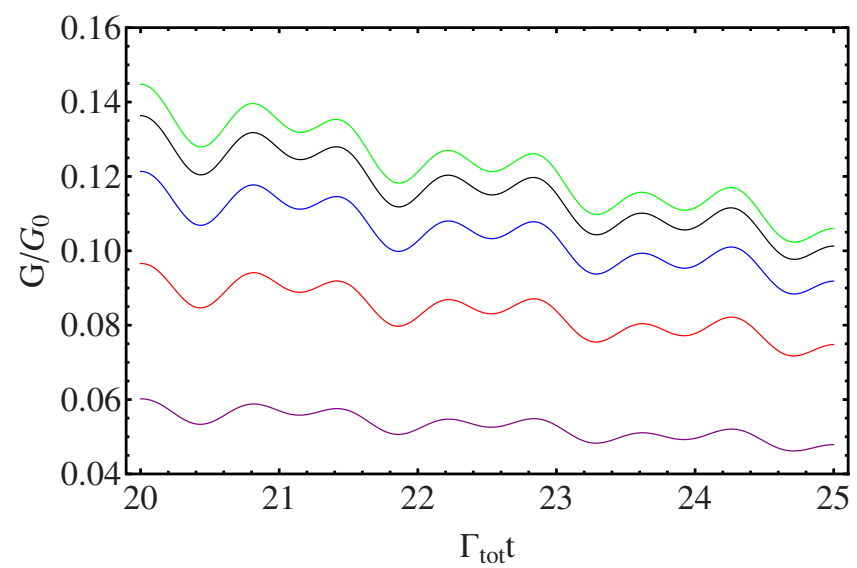

FIG. 4. (Color online) The green, black, blue, red, and purple curves (from top to bottom) represent the instantaneous conductance versus time in the Kondo time scale after the dot level has been switched to its final position for an asymmetry factor of 0.9 at $T=0.0015 \Gamma_{t o t}, \quad T=0.0030 \Gamma_{t o t}, \quad T=0.0060 \Gamma_{t o t}, \quad T=0.0090 \Gamma_{t o t}$, and $T=0.0150 \Gamma_{t o t}$, respectively, for constant $\Gamma_{t o t}$ with a bias of $V=T_{K}$. 
the sharp features in the gold density of states located at -2.08 and $-4.46 \mathrm{eV}$. As the electrons in the contacts are assumed to be noninteracting, the density of states of the contact is time independent and its sharp features are static. On the other hand, the Kondo resonance is part of the density of states of the dot. Consequently, its formation is time dependent and dynamical, which leads to persistence of the beating behavior until the Kondo resonance is fully formed. The ratio of $\omega_{1}$ to $\omega_{2}$ supports this scenario.

Moreover, the reduction in the oscillation amplitudes with increasing source-drain bias is due to the fact that the Kondo resonance starts getting destroyed and interference vanishes. The saturation of the oscillation amplitudes below $T_{K}$ is a further testimony to the proposed mechanism since the Kondo resonance is best developed below this scale. Hence, the interference strength stays the same. We note that the validity of our results in the transient regime can be checked by considering a second system with a smaller $T_{K}$. This can be accomplished by taking a slightly lower final dot level $\epsilon_{d o t}$. The time-dependent conductance curves overlap for both systems as a function of $T_{K} t$. This proves that our calculations obey universality, which is a hallmark of the Anderson model.

In conclusion, we have studied the transient current in a single electron transistor consisting of gold contacts in response to an abrupt switching of its dot level. We have used the density of states of gold, as obtained from $a b$ initio electronic structure calculations, as input in a many-body calculation. We find that an asymmetrically coupled system exhibits complex oscillations in the Kondo time scale. The two distinct frequencies that give rise to the observed beating behavior are found to be proportional to the separation between the Fermi level and the two sharp features in the density of states. We interpret this as interference between the emerging Kondo resonance and the van-Hove singularities. We note that more frequencies may mix with the two dominant frequencies in an actual experiment due to additional features in the gold density of states, see Fig. 1. However, their effects on the reported findings would not be discernible as the amplitudes are negligible as compared to those captured by our fitting.

Finally, we feel that the situation discussed in this Rapid Communication can be realized with today's experimental technology since ultrafast pump-probe experiments can capture the picosecond time scale. ${ }^{25}$ This is close to the time needed for forming the Kondo resonance. ${ }^{4}$ It would be greatly desirable to confirm our predictions experimentally.
${ }^{1}$ I R Committee, International Technology Roadmap for Semiconductors, Japan Electronics and Information Technology Industries Association, Tokyo, 2004.

${ }^{2}$ J. M. Elzerman, R. Hanson, L. H. W. van Beveren, B. Witkamp, L. M. K. Vandersypen, and L. P. Kouwenhoven, Nature (London) 430, 431 (2004).

${ }^{3}$ G. Feve, A. Mahe, J. M. Berroir, T. Kontos, B. Placais, D. C. Glattli, A. Cavanna, B. Etienne, and Y. Jin, Science 316, 1169 (2007).

${ }^{4}$ P. Nordlander, M. Pustilnik, Y. Meir, N. S. Wingreen, and D. C. Langreth, Phys. Rev. Lett. 83, 808 (1999).

${ }^{5}$ M. Plihal, D. C. Langreth, and P. Nordlander, Phys. Rev. B 61, R13341 (2000).

${ }^{6}$ A. Schiller and S. Hershfield, Phys. Rev. B 62, R16271 (2000).

${ }^{7}$ J. Merino and J. B. Marston, Phys. Rev. B 69, 115304 (2004).

${ }^{8}$ M. Plihal, D. C. Langreth, and P. Nordlander, Phys. Rev. B 71, 165321 (2005).

${ }^{9}$ F. B. Anders and A. Schiller, Phys. Rev. Lett. 95, 196801 (2005).

${ }^{10}$ F. B. Anders and A. Schiller, Phys. Rev. B 74, 245113 (2006).

${ }^{11}$ A. F. Izmaylov, A. Goker, B. A. Friedman, and P. Nordlander, J. Phys.: Condens. Matter 18, 8995 (2006).

${ }^{12}$ A. Goker, B. A. Friedman, and P. Nordlander, J. Phys.: Condens. Matter 19, 376206 (2007).

${ }^{13}$ E. Gull, P. Werner, O. Parcollet, and M. Troyer, EPL 82, 57003 (2008).
${ }^{14}$ P. Werner, T. Oka, and A. J. Millis, Phys. Rev. B 79, 035320 (2009).

${ }^{15}$ T. L. Schmidt, P. Werner, L. Mühlbacher, and A. Komnik, Phys. Rev. B 78, 235110 (2008).

${ }^{16}$ Y. Zhu, J. Maciejko, T. Ji, H. Guo, and J. Wang, Phys. Rev. B 71, 075317 (2005).

${ }^{17}$ J. Maciejko, J. Wang, and H. Guo, Phys. Rev. B 74, 085324 (2006).

${ }^{18}$ L. H. Wang, Y. Guo, C. F. Tian, X. P. Song, and B. J. Ding, J. Appl. Phys. 107, 103702 (2010).

${ }^{19}$ P. Blaha, K. Schwarz, G. K. H. Madsen, D. Kvasnicka, and L. Luitz, WIEN2K, An Augmented PlaneWave +Local Orbitals Program for Calculating Crystal Properties (Techn. Universität, Wien, 2001).

${ }^{20}$ U. Schwingenschlögl and C. Schuster, Phys. Rev. Lett. 102, 227002 (2009); Chem. Phys. Lett. 449, 126 (2007).

${ }^{21}$ J. P. Perdew, K. Burke, and M. Ernzerhof, Phys. Rev. Lett. 77, 3865 (1996).

${ }^{22}$ H. Shao, D. C. Langreth, and P. Nordlander, Phys. Rev. B 49, 13929 (1994).

${ }^{23}$ A. P. Jauho, N. S. Wingreen, and Y. Meir, Phys. Rev. B 50, 5528 (1994).

${ }^{24}$ J. Kondo, Prog. Theor. Phys. 32, 37 (1964).

${ }^{25}$ Y. Terada, S. Yoshida, O. Takeuchi, and H. Shigekawa, J. Phys.: Condens. Matter 22, 264008 (2010). 